\title{
Randomly Amplified Polymorphic DNA Fingerprinting for Identifying Rabbiteye Blueberry (Vaccinium ashei Reade) Cultivars
}

\author{
Myneni Aruna, Max E. Austin, and Peggy Ozias-Akins ${ }^{1}$ \\ Department of Horticulture, University of Georgia Coastal Plain Experiment Station, Tifton, GA 31793
}

Additional index words. PCR

\begin{abstract}
Cultivars of the economically important rabbiteye blueberry (Vaccinium ashei Reade) were differentiated at the DNA level using the technique of randomly amplified polymorphic DNA. Single decanucleotide primers of arbitrary sequence were used to amplify genomic DNA by the polymerase chain reaction. All cultivars tested exhibited a unique set of collective amplified fragments of distinct molecular weight. A blind fingerprinting experiment resulted in identification of unknown samples without ambiguity. We also clarified the genetic identity of two wild selections of rabbiteye blueberry, 'Ethel' and 'Satilla', which have been maintained as two different selections, hut are considered by some blueberry breeders to be of the same genetic constitution. The technique also verified the probable identity of two cultivars in a commercial blueberry field by comparing their amplified DNA patterns with those of standard cultivars. No variation was observed between the amplification profiles of 'Brightwell' and its presumed sport. A cultivar key based on 11 markers amplified by four primers is presented.
\end{abstract}

Cultivars of rabbiteye blueberry generally have been identified by morphological descriptors. In spite of the fact that blueberry cultivars are clonally propagated, and thus assumed to be genetically identical, some degree of morphological variation due to plant age or growing conditions may occur. It is not unusual for commercial growers and breeders to have difficulty accurately identifying plants whose clonal origin is questionable. Proper cultivar/germplasm identification is requisite in commercial plantings and breeding nurseries of this cross-pollinated species to insure high crop yields and to optimize germplasm maintenance and utilization. The current study focused on fingerprinting nineteen rabbiteye blueberry cultivars by DNA amplification with single primers of arbitrary sequence using the polymerase chain reaction [arbitrarily-primed polymerase chain reaction (AP-PCR, Welsh and McClelland, 1990) randomly amplified polymorphic DNA (RAPD) Williams et al., 1990]. We have shown in a previous study that RAPD fragments can be used to determine the genetic relatedness among rabbiteye blueberry cultivars (Aruna et al., 1993), an approach that might be useful for expanding the genetic base of cultivated genotypes.

The principal objective of the present study using RAPD analysis of rabbiteye blueberry cultivars was to develop a cultivar identification key based on amplification fragments generated by a minimal number of primers, and to apply this information to the analysis of unknown samples for cultivar identification.

\section{Materials and Methods}

Plant material. Plant material was collected from the Blueberry Research Farm, Alapaha, Georgia, the Coastal Plain Experiment Station (CPES), Tifton, Georgia, two commercial fields at Alma, Georgia, and a plantation near Thomasville, Geogia ('Satilla').

Received for publication 22 Nov. 1994. Accepted for publication 11 May 1995. This work was funded by a grant from the Georgia Seed Development Commission and from state and Hatch funds allocated to the Georgia Agricultural Experiment Station. We thank Sammy Mason for his assistance in collection of plant material. The cost of publishing this paper was defrayed in part by the payment of page charges. Under postal regulations, this paper therefore must be hereby marked advertisement solely to indicate this fact.

'To whom reprint requests should be addressed.
Fingerprinted genotypes included four wild selections, 'Ethel' (E), 'Clara' (CL), 'Myers' (MR), 'Black Giant' (BG), and 15 improved cultivars, viz., 'Callaway' (CW), 'Climax' (CM), 'Woodard' (W), 'Briteblue' (BB), 'Tifblue' (TB), 'Homebell' (H), 'Menditoo' (MD), 'Coastal' (CS), 'Bluegem' (BM), 'Premier' (PR), T-15 (T15), 'Bluebelle' (BL), 'Brightwell' (BW), 'Powderblue' (PB). and 'Delite' (D). For the blind fingerprinting experiment, three samples (B1, B2, and B3), known to be from the above 19 cultivars but whose exact identities were not disclosed to the researchers, were tested.

Experiment I. To differentiate 'Satilla' (S) from 'Ethel' (E), two clones of 'Ethel' (E1 and E2) and one of 'Satilla' were tested. 'Callaway' (CW) was used as a positive control for an alternative amplification pattern. Initially, four 'Ethel' clones were tested, but in later trials only two 'Ethel' clones were compared with 'Satilla' since no intra-clonal variation was observed.

Experiment II. Anonymous material (U1 and U2) was collected from commercial fields in Alma along with known 'Tifblue' clones from each respective field (TB2 from same field as U1, TB3 from same field as U2). A third' Tifblue' sample, TB1, was collected from the CPES. 'Brightwell' was tested along with the above material, since some of the unidentified material morphologically resembled 'Brightwell'. 'Ethel' and 'Clara', parents of 'Tifblue', were also included in this experiment.

Experiment III. Two clones (M1 and M2) of a morphological variant of the cultivar Brightwell, which is presumed to be a sport, were compared with two clones of 'Brightwell' (BW1 and BW2). The sport had been detected as an off-type branch on the cultivar Brightwell. It was later clonally propagated and maintained as a separate line. The clones of the sport were consistently early maturing over several years and could be easily differentiated visually from 'Brightwell'. 'Tifblue' and 'Menditoo', parents of 'Brightwell', also were included in this experiment.

DNA amplification. Genomic DNA was extracted from young leaves that were not yet fully expanded by a modified procedure of Bernatzky and Tanksley (1986) as described in Aruna et al. (1993). Decanucleotide primers, OPC12, OPC17, and OPG01 to OPG20' (Operon Technologies, Alameda, Calif.), were used. Experiments were repeated 3-5 times with primers OPG02, OPG05, OPG09, and OPG12. Amplification reaction conditions were essentially as 
reported by Williams et al. (1990). Genomic DNA (25 ng) was amplified\& a $25-\mu \mathrm{l}$ reaction mixture consisting of $10 \mathrm{~mm}$ Tris- $\mathrm{HCl}$ (pH 9.0), $50 \mathrm{~mm} \mathrm{KC1,} 1.54 \mathrm{~mm} \mathrm{MgCl}_{2}, 0.1 \%$ Triton X-100, $100 \mu \mathrm{M}$ each of dATP, dTTP, dCTP, dGTP and $1.25 \mathrm{U}$ Taq DNA Polymerase (Promega, Madison, Wis.). DNA was denatured at 94C for $1 \mathrm{~min}$, annealed at $36 \mathrm{C}$ for $1 \mathrm{~min}$, and extended for $2 \mathrm{~min}$ at $72 \mathrm{C}$ for 35 cycles in a DNA thermal cycler (Perkin Elmer, Norwalk, Conn.). $20 \mu \mathrm{l}$ of amplified product was electrophoresed in $2 \%$ agarose gels. The amplified products were visualized after staining with ethidium bromide. Amplified bands were scored from the film negatives.

Southern blotting and hybridization. Amplified DNA fragments from primers OPG02 and OPG09 were blotted onto hybridization membrane (GeneScreen Plus; DuPont/NEN, Boston) by capillary action (Southern, 1975). The OPG02 RAPD blot was probed with either the entire mixture of amplified fragments ('Climax' amplified with OPG02) or a gel-purified 450 bp fragment from 'Coastal' amplified with OPG02. The OPG09 RAPD blot was probed with the entire mixture of OPG09-amplified fragments from blind sample, B 1 . The $450 \mathrm{bp}$ OPG02-amplified fragment from 'Coastal' was gel purified using a DNA Purification System (Wizard PCR Preps; Promega). Labeling of fragments was carried out with $\left[\alpha-{ }^{32} \mathrm{P}\right]$-dCTP by random hexamer priming (Primea-Gene, Promega). Hybridization was performed in a hybridization incubator (Robbins Scientific, Sunnyvale, Calif.) at $65 \mathrm{C}$ for $16 \mathrm{~h}$. Blots were washed for $5 \mathrm{~min}$ at room temperature with $2 \times$ $\mathrm{SSC}+1 \%$ SDS $(20 \times \mathrm{SSC}=3 \mathrm{MNaCl}, 0.3 \mathrm{~m}$ sodium citrate, $\mathrm{pH} 7.5)$ followed by two washes of $30 \mathrm{~min}$ each at $65 \mathrm{C}$, the first with $0.5 \times$ SSC $+1 \%$ SDS and the second with $0.1 \times$ SSC $+0.1 \%$ SDS. Blots were exposed to Kodak X-OMAT film for $24 \mathrm{~h}$ at $-80 \mathrm{C}$.

\section{Results and Discussion}

RAPD patterns and cultivar key. The collective amplification profiles were highly informative and provided a unique fingerprint of each cultivar (Figs. $1 \mathrm{~A}$ and B, 2, and 3). Amplifications generally were reproducible over time and resulted in the same RAPD profiles. Four primers were tested several times to examine the reproducibility of the amplification reactions. Only one monomorphic fragment in the profile produced by primer OPG09 failed to amplify in one of five trials. The homology of similarly sized RAPD bands was confirmed in only one experiment by hybridization of the $450 \mathrm{bp}$ fragment (OPG02) from one of the cultivars only to the same-sized molecular weight fragment from all the other cultivars and the three blind samples (Fig. 1C). Labeled mixtures of amplified fragments from one cultivar (either 'Climax' or B 1) detected only those bands that were in common between the probe cultivar and other cultivars after DNA/DNA hybridization.

In the blind fingerprinting experiment using primer OPG02, the amplification phenotype of the first blind sample matched with that of 'Delite' (Fig. 1A, lanes B1 and D). The second and third blind samples had the same RAPD pattern as 'Bluebelle' (Fig. 1 A, lanes B2, B3, and BL). After tentatively identifying B 1 as 'Delite' and B2 and B3 as 'Bluebelle', we compared these samples (B1, B2, and B3) again with 'Delite', 'Bluebelle' and their parents using the same primer (Fig. 1B). The amplification patterns absolutely matched, and we later confirmed the identities of these samples from the individual who collected them.

A key was developed for 19 cultivars using two primers, OPC12 and OPC17, selected from our previous study (Aruna et al., 1993) because of their clear amplification patterns, and two additional primers, OPG09 and OPG12. Based on the presence of three RAPD bands amplified by OPC17 (OPC17-700, 1400, and 1800)
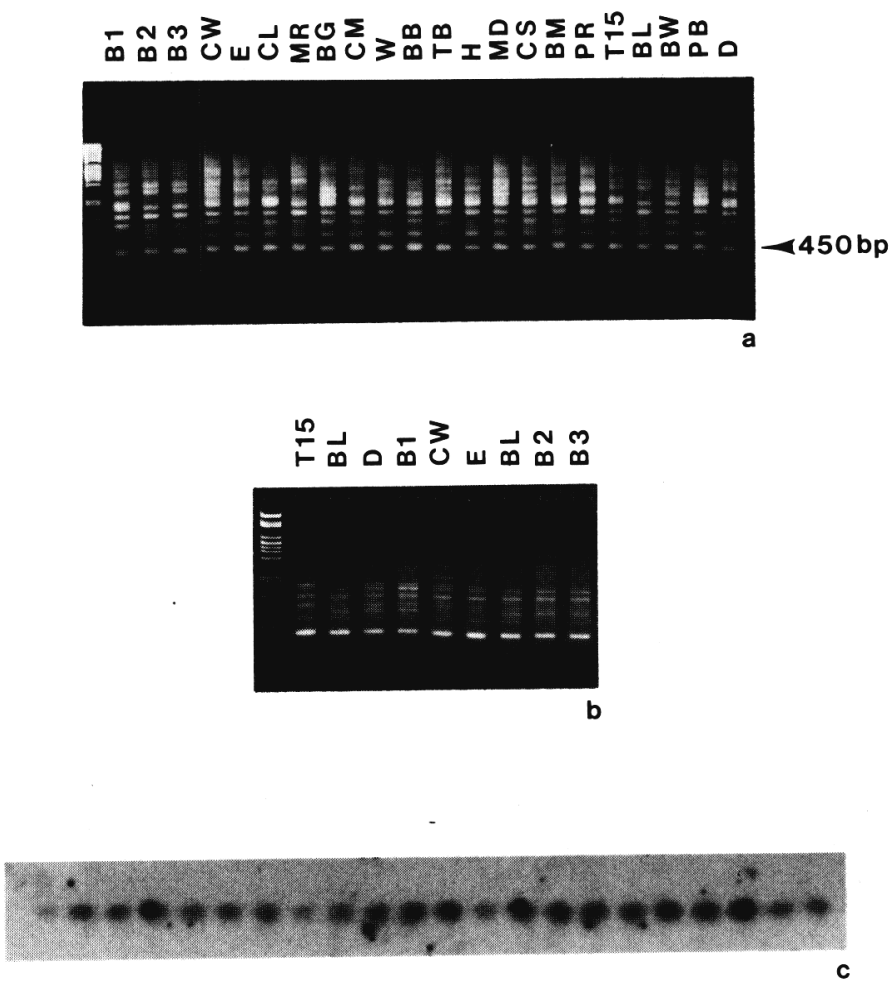

Fig. 1. DNA amplification fingerprints of the blind samples, wild selections, and cultivars of rabbiteye blueberry amplified with primer OPG02 (A and B). Abbreviations are given in Materials and Methods. (C) Hybridization of gelpurified OPG02-450 to blot of gel shown in A. Unlabeled lanes are lambda DNA digested with PstI.

(Fig. 2A), cultivars were subdivided into three groups. All three of these fragments were amplified in three cultivars (group I), two fragments (OPC17-700, 1400) were amplified in nine of the cultivars (group II) and only one fragment (OPC17-700) was amplified in seven cultivars (group III). Since OPC17-700 was amplified in all cultivars, it served as a positive control for PCR amplification. Cultivars within groups I, II, and III could be further differentiated by fragments amplified by OPC 12 (Fig. 2B), OPG12 (Fig. 2C), and OPG09 (Fig. 3). A key was developed using only 11 selected fragments OPC17-700, OPC17-1400, OPC17-1800, OPC12-730, OPC12-1090, OPG12-480, OPG12-725, OPG121000, OPG12-1100, OPG09-720, and OPG09-600 (Fig. 4).

The ability to identify unknowns and distinguish cultivars, the reproducibility of the amplification patterns, the absence of clonal variation and the apparent homologous nature of similarly-sized RAPD bands indicate that this technique may be practically applied for rabbiteye blueberry cultivar identification. The RAPD phenotypes of closely related cultivars displayed sufficient polymorphism to allow us to unambiguously identify three unknowns taken from a set of known cultivars. However, cultivar identification by comparison of all available genotypes would be expensive and time consuming. To reduce the number of comparisons required to identify a genotype, we have developed a hierarchical key using 11 RAPD bands amplified by 4 primers to distinguish four wild rabbiteye blueberry selections and 15 improved cultivars which represent most of the commercially cultivated genotypes. The limitation to the use of this key is the unknown banding patterns of cultivars or breeding lines we did not survey. It is possible that closely related genotypes might not be distinguished by this subset of RAPD markers, although our evidence indicates that cultivars with common pedigrees can be differentiated from one another (Aruna et al., 1993). Our previous 

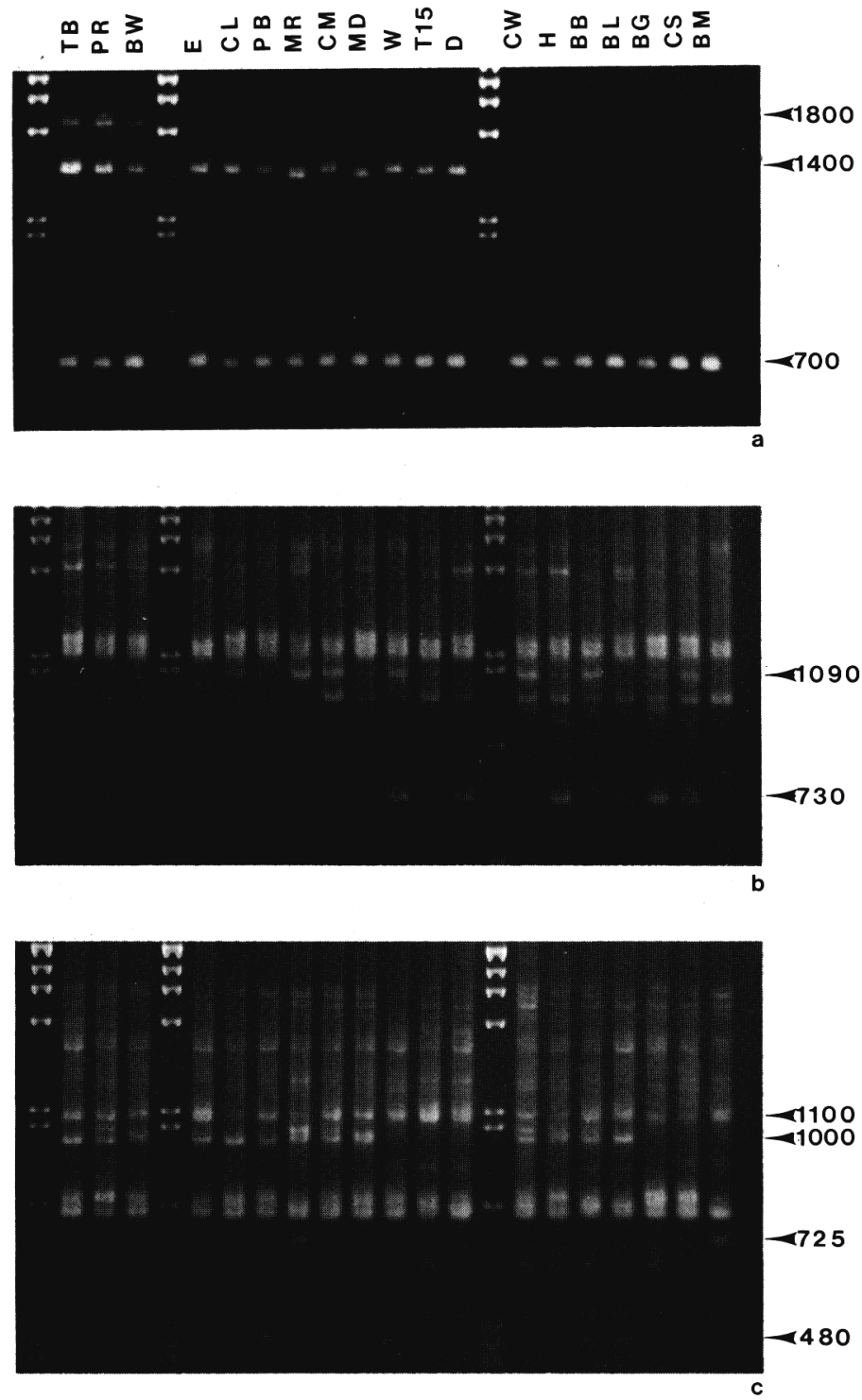

Fig. 2. Amplification profiles from primers OPC17 (A), OPC12 (B), and OPG12 (C) used in developing the key. Abbreviations are given in Materials and Methods. Unlabeled lanes are lambda DNA digested with PstI.

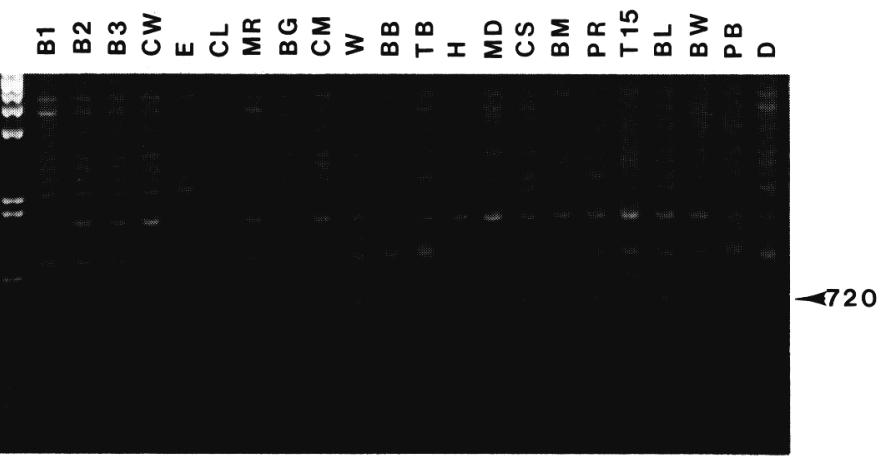

Fig. 3. Amplification profiles from primer OPG09 used in developing the key. Abbreviations are given in Materials and Methods. Unlabeled lane is lambda DNA digested with PstI.

study also showed that genetic inferences, i.e., parent-progeny relationships based on band sharing, could be made using RAPD data.

RAPDs have been applied as germplasm descriptors and have been reported to identify cultivars of many crop plants (Newbury
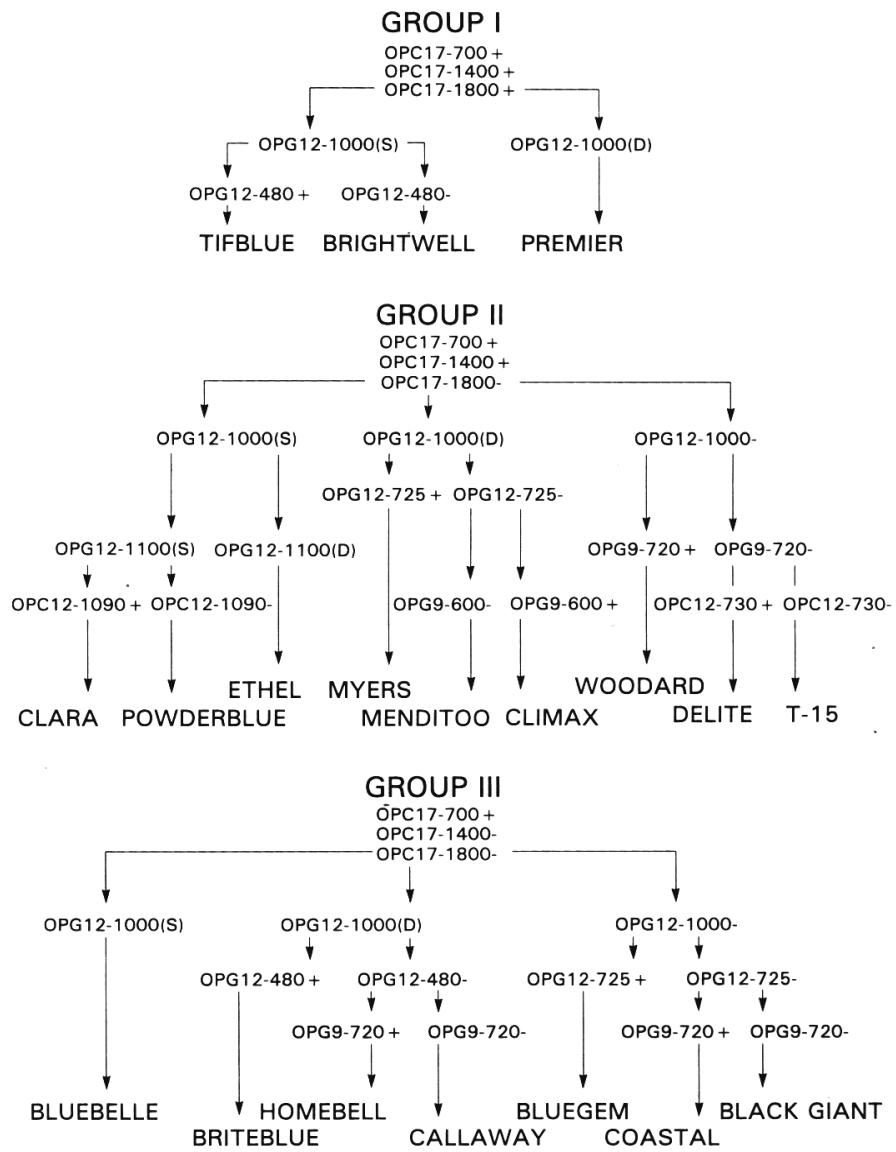

Fig. 4. Cultivar key for 19 rabbiteye blueberry cultivars based on RAPD fragments amplified using primers OPC17, OPC12, OPG09 and OPG12: Group I (A), Group II (B), and Group III (C). (+) Band present, (-) band absent, (D) double band, (S) single band.

and Ford-Lloyd, 1993). The polymorphisms revealed by RAPD markers differ from crop to crop and the number of primers necessary to differentiate test material also varies. Broccoli and cauliflower could be distinguished by using only two and three arbitrary primers, respectively (Hu and Quiros, 1991), and only 2 primers were necessary to distinguish 18 chrysanthemum cultivars (Wolff and Rijn, 1993). Koller et al. (1993) developed a key for 11 apple cultivars based on 14 RAPD markers amplified by 2 primers. For the genotypes in our study, the banding patterns obtained with most of the primers were complex and only some bands were unique. The most reproducible and unambiguous bands were used to produce the cultivar key.

Experiment I. The RAPD profiles of both samples of 'Ethel' matched with those of 'Satilla' but not 'Callaway' for all primers, OPG01 to OPG19. In all, 186 bands generated by the 19 primers were compared between 'Ethel', 'Satilla', and 'Callaway' of which 111 bands were polymorphic between 'Ethel'/'Satilla' and 'Callaway'.

Initial rabbiteye blueberry selections for breeding programs were made based on superior plant and fruit characteristics. 'Ethel' has been widely used in most of the rabbiteye blueberry cultivar improvement programs. Our results indicated that 'Satilla' and 'Ethel' likely were clonal selections derived from the same individual, supporting the suggestion of some rabbiteye breeders that the two names, 'Satilla' and 'Ethel', had been assigned to a single selection (Austin, 1984). 
Experiment II. The two unknowns from Alma (U1 and U2) were both identified by their RAPD profiles as 'Tifblue' based on 155 polymorphic fragments out of a total of 213 fragments amplified in this experiment. The three 'Tifblue' clones tested (TB1, TB2, TB3) and the two unknowns (U1 , U2) exhibited identical RAPD profiles with 19 primers. The RAPD profiles of TB1, TB2, TB3, U1, and U2 were distinguishable from the RAPD profiles of the parents of 'Tifblue', 'Ethel' and 'Clara', and also from the cultivar, Brightwell, which was morphologically similar to the unknowns. The primers OPG07 to OPG13 and OPG15 to OPG20 could distinguish between 'Ethel', 'Clara', 'Tifblue' and unknowns, and 'Brightwell'. Fragments amplified with primer OPG02 were monomorphic in this experiment. Thus, our results were applied to resolve a cultivar identity problem encountered by commercial blueberry growers in Alma, Georgia. Two plants of questionable identity (U1 and U2) had been found among a planting of 'Tifblue'. Morphologically, the material resembled the cultivar Brightwell; however, our present study distinguished U1 and U2 as 'Tifblue' and not 'Brightwell'.

Experiment III. Using 18 RAPD primers (OPG1 to 14 and 16 to 19) which generated 137 polymorphic fragments out of a total of 222, we were unable to differentiate 'Brightwell' from clones (M1 and M2) of its supposed sport. However, all the 'Brightwell' clones could be separated from the parents, 'Menditoo' and 'Tifblue'. The primers identified three RAPD profiles in this experiment, those of 'Menditoo', 'Tifblue', and 'Brightwell', thus the cultivar Brightwell and its presumed sport (clones Ml and M2) appear to be identical. A somatic mutation could have generated this off-type, although its detection in a hexaploid plant should be rare. Single-gene mutations among the sports of 'Red Delicious' apples also were undetectable by DNA fingerprinting using a minisatellite DNA probe (Nybom, 1990). In contrast, RAPDs detected polymorphisms between two near isogenic lines of soybean, wild type and an ethyl methane sulfonate-induced mutant, after digesting DNA with restriction endonucleases prior to DNA amplification (Caetano-Anolles et al., 1993). However, it is not known whether predigesting the DNA in these samples affected the polymorphism detected or if the ethyl methane sulfonate mutagenesis resulted in major changes in the DNA. The probability of detecting a small mutational change using RAPDs or any other fingerprinting technique should be low.

To realize fully the potential of the RAPD technique for fingerprinting, primers, which can unambiguously amplify polymorphisms among the cultivars to be examined, must be identified. Some cultivars of rabbiteye blueberry differ by only one or two fragments, and it has been necessary to use several primers to distinguish these closely related genotypes. Due to the sensitivity of the RAPD technique to minor protocol changes, more transferable markers for DNA fingerprinting might be obtained from cloned RAPD fragments that have been converted into sequence characterized amplified regions (SCARs), (Lubbers et al., 1994; Paran and Michelmore, 1993) or from sequence-tagged microsatellite sites (Beckmann and Soller, 1990). Any of these alternative markers would share the advantage of targeted amplification of known sequences as well as the disadvantage of increased expense for developing the appropriate markers. The present key for rabbiteye blueberry cultivars, based on RAPD fragments, lends itself to practical application, and we conclude that for comparisons of individuals or cultivars, the technique can be routinely applied, with proper precautions, for varietal identification.

\section{Literature Cited}

Aruna, M., P. Ozias-Akins, M.E. Austin, and G. Kochert. 1993. Genetic relatedness among rabbiteye blueberry (Vaccinium ashei) cultivars determined by DNA amplification using single primers of arbitrary sequence. Genome 36:971-977.

Austin, M.E. 1984. Origin of names for original rabbiteye blueberry cultivars. Proc. 5th North Amer. Blueberry Res. Work. Conf., Univ. of Florida, Gainesville, Fla., 1-3 Feb. 1984. IFAS press, Univ. of Florida. p. 66-69.

Beckmann, J.S. and M. Soller. 1990. Toward a unified approach to genetic mapping of eukaryotes based on sequence tagged microsatellite sites. BioiTechnology 8:930-932.

Bernatzky, R. and S.D. Tanksley. 1986. Genetics of actin related sequences in tomato. Theor. Appl. Genet. 72:314-321.

Caetano-Anolles, G., B.J. Bassam, and P.M. Gresshoff. 1993. Enhanced detection of polymorphic DNA by multiple arbitrary amplicon profiling of endonuclease-digested DNA: Identification of markers tightly linked to the supernodulation locus in soybean. Mol. Gen. Genet. 241:57-64.

$\mathrm{Hu}$, J. and C.F. Quiros. 1991. Identification of broccoli and cauliflower cultivars with RAPD markers. Plant Cell Rpt. 10:505-551.

Koller, B., A. Lehmann, J.M. McDermott, and C. Gessler. 1993. Identification of apple cultivars using RAPD markers. Theor. Applied Genet. 85:901-904.

Lubbers, E.L., L. Arthur, W.W. Hanna, and P. Ozias-Akins. 1994. Molecular markers shared by diverse apomictic Pennisetum species. Theor. Applied Genet. 89:636-642.

Newbury, H. J. and B.V. Ford-Lloyd. 1993. The use of RAPD for assessing variation in plants. Plant Growth Regulat. 12:43-51.

Nybom, H. 1990. DNA fingerprints in sports of 'Red Delicious' apples. HortScience 25: 1641-1642.

Paran, I. and R.W. Michelmore. 1993. Development of reliable PCRbased markers linked to downy mildew resistance genes in lettuce. Theor. Applied Genet. 85:985-993.

Southern, E.M. 1975. Detection of specific sequences among DNA fragments separated by gel electrophoresis. J. Mol. Biol. 98:503-517.

Welsh, J. and M. McClelland. 1990. Fingerprinting genomes using PCR with arbitrary primers. Nucleic Acids Res. 18(24):7213-7218.

Williams, J.G.K., A.R. Kubelik., K.J. Livak., J.A. Rafalski, and S.V. Tingey. 1990. DNA polymorphisms amplified by arbitrary primers are useful as genetic markers. Nucleic Acids Res. 18(22):6531-6535.

Wolff, K. and J.P. Rijn. 1993. Rapid detection of genetic variability in Chrysanthemum (Dendranthema grandiflora Tzvelev) using random primers. Heredity 71:335-341. 